\title{
A CONDITION IN FINITE SOLVABLE GROUPS RELATED TO CYCLIC SUBGROUPS
}

\author{
D. IMPERATORE ${ }^{\bowtie}$ and MARK L. LEWIS
}

(Received 13 June 2010)

\begin{abstract}
In this paper, we classify the finite groups belonging to the class of cyclic-transitive groups. A group $G$ is said to be cyclic-transitive if the following condition holds: if $x, y, z$ are nonidentity elements of $G$ such that $\langle x, y\rangle$ and $\langle y, z\rangle$ are both cyclic, then $\langle x, z\rangle$ is also cyclic.
\end{abstract}

2000 Mathematics subject classification: primary 20D10.

Keywords and phrases: finite solvable groups, finite nonsolvable groups, cyclic subgroups, partitioned groups.

\section{Introduction}

A nontrivial group $G$ is said to be cyclic-transitive if generating a cyclic subgroup gives rise to a transitive relation on the set of nonidentity elements of $G$. In particular, a group $G$ belongs to the class of cyclic-transitive groups if the following condition holds: if $x, y, z$ are elements of $G \backslash\{1\}$ such that $\langle x, y\rangle$ and $\langle y, z\rangle$ are both cyclic, then $\langle x, z\rangle$ is also cyclic.

This property can be introduced as an analogue of another group-theoretical property. According to [3], if $\mathfrak{X}$ is a group-theoretical class, a group $G$ is called $\mathfrak{X}$-transitive if the conditions $\langle x, y\rangle \in \mathfrak{X}$ and $\langle y, z\rangle \in \mathfrak{X}$ imply that $\langle x, z\rangle \in \mathfrak{X}$ for all nontrivial elements $x, y, z \in G$. In a similar fashion, the class of $\mathfrak{X}$-transitive groups can be introduced in graph theoretical terms. If $G$ is a group, we can consider the graph denoted by $\Gamma_{\mathfrak{X}}(G)$ and defined in the following way: the set of vertices of $\Gamma_{\mathfrak{X}}(G)$ is the set of all nonidentity elements of $G$; two distinct vertices $x$ and $y$ are joined if they generate a subgroup belonging to $\mathfrak{X}$. It is clear that $G$ is $\mathfrak{X}$-transitive if and only if every connected component of $\Gamma_{\mathfrak{X}}(G)$ is a complete graph. There are many results concerning $\mathfrak{X}$-transitive groups for particular classes $\mathfrak{X}$.

If $\mathfrak{X}$ is the class of all abelian groups, $\mathfrak{X}$-transitive groups are also called commutative-transitive groups (or $C T$-groups for short). Groups with this property have been investigated by several authors (see, for instance, [4, 9-11]).

(C) 2010 Australian Mathematical Publishing Association Inc. 0004-9727/2010 \$16.00 
The first results on CT-groups are due to Weisner [10]; in particular, he proved that finite groups with this property are either solvable or simple. Suzuki [9] classified finite nonabelian simple groups. The structure of finite solvable CT-groups has been investigated by $\mathrm{Wu}[11]$. Furthermore, Delizia et al. (see [2, 3]) investigated the structure of $\mathfrak{X}$-transitive groups, when $\mathfrak{X}$ is a group-theoretical class satisfying the following conditions: $\mathfrak{X}$ is subgroup closed, it contains all finite abelian groups and is bigenetic in the class of all finite groups. We recall that if $\mathcal{P}$ is a group-theoretical property and $\mathfrak{X}$ is a class of groups, then $\mathcal{P}$ is said to be a bigenetic property of $\mathfrak{X}$-groups ( $\mathcal{P}$ is bigenetic in $\mathfrak{X}$-groups) if an $\mathfrak{X}$-group $G$ has the property $\mathcal{P}$ whenever all of its two-generator subgroups have $\mathcal{P}$. Such classes have also been investigated by Lennox in [7].

Finally, we mention that the cyclic graph associated with a nontrivial group $G$ has been studied in [6]; this is essentially the graph $\Gamma_{\mathfrak{X}}(G)$, where $\mathfrak{X}$ is the class of all cyclic groups. Obviously $G$ is cyclic-transitive if and only if every connected component of the this graph is a complete graph. A full description of nilpotent and supersolvable groups that are cyclic-transitive has been given in [6].

The main purpose of this paper is to classify finite groups that are cyclic-transitive. In Section 2, we show that every cyclic-transitive group can be seen as a partitioned group. More precisely, in Theorem 2.2, we prove that $G$ is cyclic-transitive if and only if it has a partition of locally cyclic subgroups. In Section 3, we quote a wellknown theorem of Suzuki that determines the structure of all nonsolvable finite groups with a nontrivial partition. Using the result of Suzuki, we obtain a characterization of nonsolvable, finite, cyclic-transitive groups (see Theorem 3.2). Finally, in the last section, we obtain Theorem 4.2 which gives a complete description of solvable, finite, cyclic-transitive groups. This result is based on Baer's classification of solvable groups with a partition.

\section{Partitioned groups}

Let $G$ be a group and let $\mathcal{A}=\left\{A_{i}\right\}_{i \in I}$ be a collection of its subgroups. Then $\mathcal{A}$ is said to be a partition of $G$ if every nonidentity element of $G$ is contained in exactly one subgroup of $\mathcal{A}$.

The subgroups $A_{i}$ in $\mathcal{A}$ are said to be the components of the partition; moreover, $\mathcal{A}$ is said to be nontrivial if every component is a proper nontrivial subgroup of $G$.

Our first result is a lemma that will be useful in proving a characterization of cyclictransitive groups in terms of partitions.

LeMma 2.1. Let $G$ be a group. Suppose that there exists a partition $\mathcal{F}$ of $G$. Then $G$ is cyclic-transitive if and only if every subgroup of $\mathcal{F}$ is cyclic-transitive.

Proof. Suppose that every subgroup of $\mathcal{F}$ is cyclic-transitive. Consider three nonidentity elements $x, y, z$ of $G$ such that $\langle x, y\rangle$ and $\langle y, z\rangle$ are both cyclic; we must show that $\langle x, z\rangle$ is cyclic. Suppose, for example, that $\langle x, y\rangle=\langle a\rangle$ and $\langle y, z\rangle=\langle b\rangle$. By hypothesis, $\mathcal{F}$ is a partition of $G$, hence there exist two subgroups $H$ and $K$ in $\mathcal{F}$ such that $\langle a\rangle \leq H$ and $\langle b\rangle \leq K$. Then $y \in\langle a\rangle \cap\langle b\rangle \subseteq H \cap K$. Since $\mathcal{F}$ is a partition, 
the only possibility is $H=K$ and therefore $\langle x, z\rangle$ is cyclic, as required. The converse is clear since the condition of being cyclic-transitive is inherited by subgroups.

Then we have the following theorem.

THEOREM 2.2. Let $G$ be a group. Then $G$ is cyclic-transitive if and only if it has a partition of locally cyclic subgroups.

Proof. Assume that $G$ is cyclic-transitive. Then we get an equivalence relation on $G \backslash\{1\}$ by saying that $a$ and $b$ are equivalent if $\langle a, b\rangle$ is cyclic. For every element $a \in G \backslash\{1\}$, let $[a]$ be the equivalence class of $a$. We claim that $[a] \cup\{1\}$ forms a subgroup of $G$. Obviously, $a^{-1} \in[a]$ since $\left\langle a, a^{-1}\right\rangle=\langle a\rangle$. If $a, b$ lie in $[a]$, then $\langle a, a b\rangle=\langle a, b\rangle$ is cyclic so either $a b=1$ or $a b \in[a]$. In any case, $a b \in[a] \cup\{1\}$. Therefore $[a] \cup\{1\}$ is a subgroup of $G$. Now, either $[a]=[b]$ or $[a] \cap[b]$ is empty. Thus, $G$ is partitioned by the subgroups given by these equivalence classes together with $\{1\}$. Moreover, $[a] \cup\{1\}$ is locally cyclic, for any $a \in G$. In fact, for every $x, y$ in $[a]$, we get that $\langle x, a\rangle$ and $\langle y, a\rangle$ are both cyclic and therefore, since $G$ is cyclictransitive, $\langle x, y\rangle$ is cyclic. Conversely, if $G$ has a partition consisting of locally cyclic subgroups, then it is cyclic-transitive by Lemma 2.1 .

\section{Nonsolvable groups}

The classification of finite groups with a nontrivial partition was proved in 1961 for solvable groups by Baer and in the general case by Suzuki. This result of Suzuki can be considered one of the most important contributions to the classification of finite simple groups, in particular in his use of character theory to show that a finite nonsolvable group with a nontrivial partition has even order.

We now state Suzuki's theorem (for the proof, the reader can refer, for instance, to [8, Theorem 3.5.11]).

THEOREM 3.1 (Suzuki). Let $G$ be a nonsolvable finite group with a nontrivial partition. Then one of the following holds:

(a) $G$ is a Frobenius group;

(b) $G \cong \operatorname{PGL}(2, q)$, $q$ a prime power, $q \geq 4$;

(c) $G \cong \operatorname{PSL}(2, q)$, $q$ a prime power, $q \geq 4$;

(d) $G \cong \operatorname{Sz}(q), q=2^{2 n+1}, n \in \mathbb{N}$.

Furthermore, as an application of Lemma 2.1, we remark that every projective special linear group of type $\operatorname{PSL}(2, q)$ is cyclic-transitive because it has a partition consisting of cyclic-transitive subgroups (see, for instance, [5, Section II.8]).

If $G=\operatorname{PGL}\left(2, p^{n}\right)$, with $p^{n}>3$, then the set of maximal cyclic subgroups is a partition (see, for instance, [5, Section II.7] or [8, Examples 3.5.1]). Consequently $G$ is cyclic-transitive, by Lemma 2.1 .

Even though the Suzuki groups are partitioned, the components of the partition are not cyclic-transitive, so the Suzuki groups are not cyclic-transitive. Hence, Lemma 2.1 and Theorem 3.1 yield the following result. 
THEOREM 3.2. Let $G$ be a nonsolvable finite group. Then $G$ is cyclic-transitive if and only if one of the following occurs:

(1) $G \cong \operatorname{PGL}(2, q), q$ a prime power, $q \geq 4$;

(2) $\quad G \cong \operatorname{PSL}(2, q)$, $q$ a prime power, $q \geq 4$.

\section{Solvable groups}

In order to study the structure of finite solvable groups that are cyclic-transitive, we need to know the structure of finite, nilpotent, cyclic-transitive groups. The first result of this section is an immediate corollary of [6, Theorem 4.1].

LEMMA 4.1. Let $G$ be a finite nilpotent group. Then $G$ is cyclic-transitive if and only if one of the following occurs:

(i) $G$ is a group of exponent p for some prime $p$;

(ii) $G$ is cyclic;

(iii) $G$ is a dihedral 2-group.

The main theorem of this section is the following. We note that the supersolvable case has previously been handled in [6, Theorem 5.2]. Appealing instead to Baer's classification of finite solvable groups with a nontrivial partition, we will not need to refer to this result in [6].

THEOREM 4.2. Let $G$ be a finite solvable group. Then $G$ is a cyclic-transitive group if and only if one of the following holds:

(1) $G$ is a cyclic group;

(2) $G$ is a p-group of exponent p for some prime $p$;

(3) $G$ is a dihedral group;

(4) $G \simeq S_{4}$;

(5) $G$ is a Frobenius group, whose complements are cyclic and whose kernel is either cyclic or of prime exponent;

(6) $G=\langle a\rangle \times H$, where $\langle a\rangle$ has prime order $p \neq 2$ and $H$ is a Frobenius group with a cyclic Frobenius kernel and Frobenius complements having order $p$.

The key to proving this is the classification of finite solvable groups with a nontrivial partition by Baer. We recall this fundamental result which appeared in [1] (for a proof, the reader can refer, for instance, to [8, Theorem 3.5.10]).

THEOREM 4.3 (Baer). Let $G$ be a finite solvable group with a nontrivial partition $\Sigma$. Then one of the following occurs:

(a) $G$ is a p-group for some prime $p$;

(b) $G$ has a nilpotent normal subgroup $N$ such that $N \in \Sigma,|G: N|$ is a prime $p$ and every element in $G \backslash N$ has order $p$;

(c) $G$ is a Frobenius group;

(d) $G \cong S_{4}$. 
Proof of TheOrem 4.2. We first suppose that $G$ is cyclic-transitive. We know by Theorem 2.2 that $G$ is partitioned by cyclic subgroups. If this partition is trivial, then $G$ is cyclic, and so we may assume that $G$ has a nontrivial partition. Thus, Baer's theorem applies. We go through the possibilities from Baer's theorem.

If $G$ is a $p$-group, then we may use Lemma 4.1 to see that $G$ is cyclic, has exponent $p$, or is a dihedral 2-group.

Next, we suppose that $G$ has a normal nilpotent subgroup $N$ of prime index $p$ where every element of $G \backslash N$ has order $p$. Notice that $N$ is now cyclic-transitive and nilpotent. Hence, $N$ is cyclic, has prime exponent, or is a dihedral 2-group. If $p$ does not divide $|N|$, then the fact that every element outside $N$ has order $p$ forces $G$ to be a Frobenius group with Frobenius kernel $N$. If $N$ is cyclic or has prime exponent, then we are done. Thus, we may assume that $N$ is a dihedral 2-group, and it is not difficult to see that a dihedral 2-group cannot be a Frobenius kernel.

Thus, we may assume that $p$ divides $|N|$. If $N$ has prime exponent or is a dihedral 2 -group, then this implies that $G$ is a $p$-group, and we are done. Thus, we may assume that $N$ is cyclic. If $p=2$, we now have exactly the condition to force $G$ to be a dihedral group. Hence, we suppose that $p$ is odd. Let $P$ be a Sylow $p$-subgroup of $G$. Now, $P$ is not cyclic, so Lemma 4.1 implies that $P$ has exponent $p$. Since $N \cap P$ is cyclic, this implies that $N \cap P=\langle a\rangle$ has order $p$. Let $x \in P \backslash(N \cap P)$, and let $H=\langle x, Q\rangle$ where $Q$ is the Hall $p$-complement of $N$. Observe that $\langle a\rangle$ centralizes both $x$ and $Q$, so $G=\langle a\rangle \times H$. Also, every element in $H \backslash Q$ lies in $G \backslash N$, and so, every element of $H \backslash Q$ has order $p$. It follows that $H$ is a Frobenius group whose Frobenius kernel is cyclic and whose Frobenius complements have order $p$.

We next suppose that $G$ is a Frobenius group. Let $N$ be the Frobenius kernel of $G$ and let $H$ be a Frobenius complement. We know $N$ is nilpotent, and so Lemma 4.1 implies that $N$ is either cyclic, has prime exponent, or is a dihedral 2-group. As already mentioned, a dihedral 2-group cannot be a Frobenius kernel, so $N$ is either cyclic or has prime exponent as desired. We know that $H$ is cyclic-transitive, so we can consider the possibilities from Baer's theorem. If $H$ is a $p$-group, then we know that $H$ is cyclic or generalized quaternion since it is a Frobenius complement. Also, by Lemma 4.1, we have that $H$ is cyclic, has exponent $p$, or is a dihedral 2-group. It follows that if $H$ is a $p$-group, then $H$ must be cyclic.

We may now suppose that $H$ is not cyclic. Earlier we classified the cyclic-transitive groups that have type (b) from Baer's classification. Either, they are a Frobenius group, a $p$-group, a dihedral group, or they have a elementary abelian Sylow $p$-subgroup of order $p^{2}$. We know that a Frobenius complement cannot be a Frobenius group. Also, it cannot have a Sylow 2-subgroup that is dihedral nor a Sylow $p$-subgroup that is elementary abelian of order $p^{2}$. Also, the Sylow 2-subgroup of $S_{4}$ is not cyclic nor generalized quaternion, so $H$ is not $S_{4}$. We conclude that $H$ is cyclic.

The final possibility is that $G$ is $S_{4}$ which is one of the conclusions.

Conversely, suppose that $G$ is one of the given groups. To show that $G$ is cyclictransitive, it suffices by Lemma 2.1 to show that $G$ has a partition by cyclic-transitive subgroups. If $G$ is cyclic, this is obvious. If $G$ has exponent $p$, then it is partitioned by the subgroups of order $p$ which are necessarily cyclic. If $G$ is a dihedral group, it is 
partitioned by the cyclic subgroup $C$ of index 2 and the subgroups of order two which intersect $C$ trivially.

Observe that $S_{4}$ is cyclic-transitive, since it is partitioned by the set of maximal cyclic subgroups (see, for instance, [8, Examples 3.5.1]). Suppose that $G$ is a Frobenius group, whose complements are cyclic and whose kernel is either cyclic or of prime exponent. We know $G$ is partitioned by its kernel and complements, and they are all cyclic-transitive.

Finally, suppose that $G=\langle a\rangle \times H$, where $\langle a\rangle$ has prime order $p \neq 2$ and $H$ is a Frobenius group with a cyclic Frobenius kernel and Frobenius complements having order $p$. Let $K$ be the Frobenius kernel of $H$ and let $N=\langle a, K\rangle=\langle a\rangle \times K$. Observe that $N$ is cyclic. Suppose that $g \in G \backslash N$. Then $g=a^{m} h$, where $m$ is an integer and $h \in H \backslash K$. Now, $a^{m}$ and $h$ commute. So the order of $g$ is the least common multiple of the orders of $a^{m}$ and $h$. But $h$ has order $p$ and $a^{m}$ has order dividing $p$, so $g$ has order $p$. We conclude that all the elements in $G \backslash N$ have order $p$, and so $G$ is partitioned by $N$ and the subgroups of order $p$ that intersect $N$ trivially. In particular, $G$ is cyclic-transitive.

\section{References}

[1] R. Baer, 'Partitionen endlicher Gruppen', Math. Z. 75 (1960/61), 333-372.

[2] C. Delizia, P. Moravec and C. Nicotera, 'Groups in which the bounded nilpotency of two-generator subgroups is a transitive relation', Beiträge Algebra Geom. 48 (2007), 69-82.

[3] C. Delizia, P. Moravec and C. Nicotera, 'Finite groups in which some property of two generator subgroups is transitive', Bull. Amer. Math. Soc. 75 (2007), 313-320.

[4] C. Delizia and C. Nicotera, 'On certain group theoretical properties generalizing commutativity', in: Proceedings of Ischia Group Theory 2004, Contemporary Mathematics, 402 (American Mathematical Society, Providence, RI, 2006), pp. 143-148.

[5] B. Huppert, Endliche Gruppen I (Springer, Berlin, 1967).

[6] D. Imperatore, 'On a graph associated with a group', in: Proceedings of International Conference Ischia Group Theory (World Scientific Publishing, Singapore, 2008), pp. 100-115; ISBN-13: 9789814277792.

[7] J. C. Lennox, 'Bigenetic properties of finitely generated hyper-(abelian-by-finite) groups', J. Aust. Math. Soc. 16 (1973), 309-315.

[8] R. Schmidt, Subgroup Lattices of Groups (Walter de Gruyter, New York, 1994).

[9] M. Suzuki, 'The nonexistence of a certain type of simple groups', Proc. Amer. Math. Soc. 8 (1957), 686-695.

[10] L. Weisner, 'Groups in which the normaliser of every element except identity is abelian', Bull. Amer. Math. Soc. 31 (1925), 413-416.

[11] Y. F. Wu, 'Groups in which commutativity is a transitive relation', J. Algebra 207 (1998), 165-181.

\section{IMPERATORE, Dipartimento di Matematica e Applicazioni 'R. Caccioppoli', Università degli Studi di Napoli 'Federico II', Via Cinthia-I 80126, Napoli, Italy e-mail: diana.imperatore@unina.it}

MARK L. LEWIS, Department of Mathematical Sciences, Kent State University, Kent, Ohio 44242, USA

e-mail: lewis@math.kent.edu 